\title{
Automatic segmentation and recognition of red and white cells in stool microscopic images of human
}

\author{
Guanghui Kong ${ }^{1, *}$, Zhiyong Wang ${ }^{1}$, Xiuchao Wan ${ }^{1}$, and Fengjun Xue ${ }^{2}$ \\ ${ }^{1}$ School of Electrical and Electronic Engineering, Tianjin University of Technology, China \\ ${ }^{2}$ Wuhan Running Education Research Institute, Wuhan, Hubei Province, China
}

\begin{abstract}
Aiming to solve the problem of low efficiency in manually recognizing the red and white cells in stool microscopic images, we propose an automatic segmentation method based on iterative corrosion with marker-controlled watershed segmentation and an automatic recognition method based on support vector machine (SVM) classification. The method first obtains saliency map of the images in HSI and Lab color spaces through saliency detection algorithm, then fuses the salient images to complete the initial segmentation. Next, we segment the red and white cells completely based on the initial segmentation images using marker-controlled watershed algorithm and other complementary methods. According to the differences in geometrical and texture features of red and white cells such as area, perimeter, circularity, energy, entropy, correlation and contrast, we extract them as feature vectors to train SVM and finally complete the classification and recognition of red and white cells. The experimental results indicate that our proposed marker-controlled watershed method can help increase the segmentation and recognition accuracy. Moreover, since it is also less susceptible to the heteromorphic red and white cells, our method is effective and robust.
\end{abstract}

\section{Introduction}

Stool microscopic image analysis is an optical image processing method for the detection of various tangible objects in stool microscopic images, in order to check if a person's internal digestive system has diseases, injuries or potential lesions [1]. In the routine examination of stool in hospitals, the number of red and white cells in stool microscopic images is one of the most clinically essential parameters taken into doctor's consideration [2] In order to make the characteristics of red and white cells more obvious, hospitals usually use staining, adding fluorescence and other ways to mark the image. However, these methods will increase the cost of detection. In this paper, we are going to introduce a detection approach based on stool microscopic images without staining or marking. The original microscopic images without straining, the red and white cells and the tangible components similar to red and white cells are shown in Fig.1.

Many image segmentation and recognition methods have been applied in the field of cell analysis in recent years. For instance, edge detection [3], threshold processing [4], watershed segmentation [5] and level set segmentation [6] are commonly used for cell segmentation, while BP neural network [7], random forest [6] and convolutional neural network [8] for recognition. In the routine examination of stool in

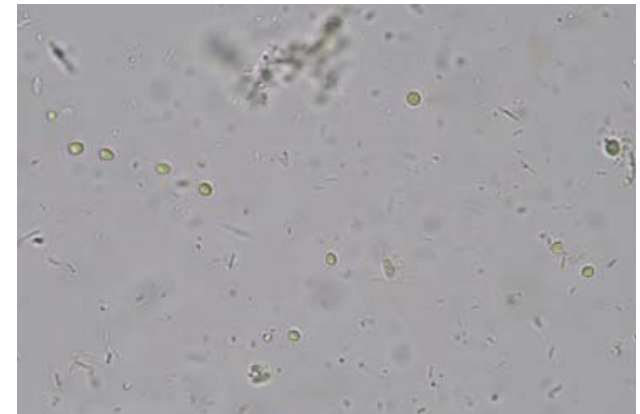

(a) Stool microscopic image

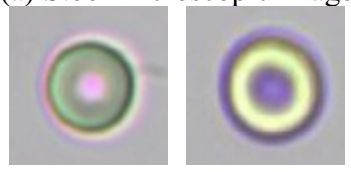

(b) Red cells

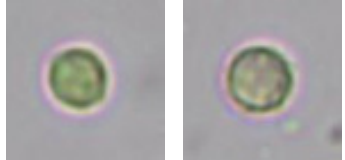

(c) White cells

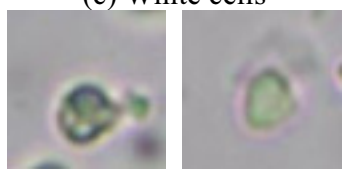

(d) Tangible component similar to red or white cells

Fig.1. Microscopic image of stool and its tangible components

hospitals, image segmentation and recognition not only require a high recognition accuracy, but also require a

\footnotetext{
*Corresponding author: 183123337@stud.tjut.edu.cn
} 
certain real-time performance to ensure the speed and efficiency of recognition. Due to the problems of blurred edges, low contrast, and unclear structures of the red and white cells, it is difficult to segment all target components in the stool microscopic images using only single segmentation method. Inspired by the advantages of some different segmentation methods, we propose a combination method based on multiple segmentation algorithms to complete the segmentation of stool microscopic images. We first use the luminance, saturation and color characteristics in the HSI and Lab color space to obtain the saliency map of the stool microscopic images [9]. Then the saliency map is fine segmented by marker-controlled watershed algorithm based on iterative corrosion. In order to mark the foreground and background of the images, we apply the extended minimum transform method (H-minima) proposed by $P$. Soille et al. [10] to extract the markers. In this case the local minimums will be limited to avoid the over-segmentation problem. In the recognition part, we select some appropriate geometric and texture features (see Section 2.2) to classify our targets and other tangible components. Support vector machine (SVM) is a general learning algorithm specially proposed for small sample learning problems. It makes the separated categories have the maximum interval by constructing an optimal classification hyperplane [11]. When the samples are non-linearly separable, we use the kernel function to map the samples to a high-dimensional space, so that the nonlinear problems can be converted into linear problems [12]. SVM is based on the principle of structural risk minimization, rather than the traditional empirical risk minimization principle, which makes the classification method can not only reduce the recognition error but also improve the generalization ability of the data, making the classification of stool microscopic images more robust [13].

\section{Approach Details}

\subsection{Image Segmentation}

Before recognizing the red and white cells, it is necessary to locate suspicious target components in the stool microscopic images and separate them from the background. For this purpose, there are two main steps, which are using the saliency detection algorithm to perform the initial segmentation, and applying the maker-controlled watershed algorithm based on iterative corrosion to further fine segment the image. The procedure of the segmentation method is shown in Fig. 2.

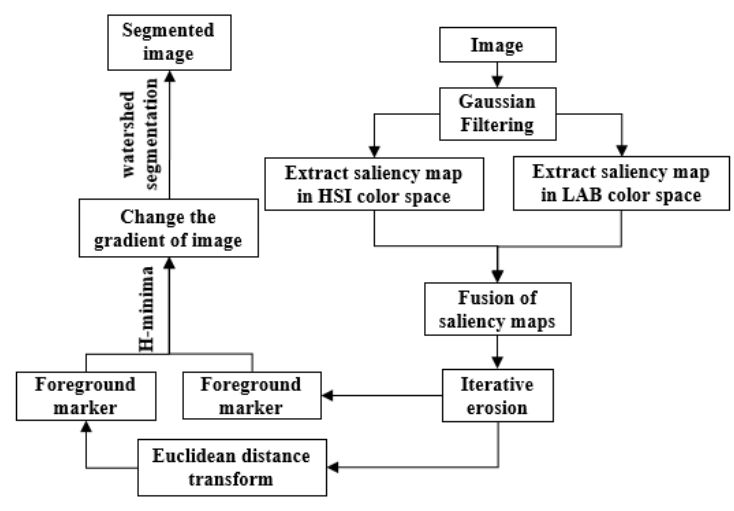

Fig.2. The flowchart of the segmentation algorithm

Firstly we extract the mean values $I_{\mu}=\left[L_{\mu}, a_{\mu}, b_{\mu}\right]^{T}$ of the three channels (luminance $(L)$ and colors ( $a$ and $b)$ ) in $L a b$ color space of the image $I$. Meanwhile we process the original image $I$ with a Gaussian filter to obtain the filtered image $I_{g}$, then convert $I_{g}$ from RGB color space to $L a b$ color space as well. We extract the three channels $L, a$ and $b$ separately, which are noted as $I_{\omega_{\text {nc }}}(x, y)=\left[L_{g}, a_{g}, b_{g}\right]^{T},\left(x, y \in R^{2}\right)$. Next, we use the Euclidean metric to fuse 3 feature matrices to obtain the saliency map. The saliency map $S$ of the image $I$ with width $W$ and height $H$ is formulated as

$$
S(x, y)=\left\|I_{\mu}-I_{\omega_{\mathrm{hc}}}(x, y)\right\|,(0<x<W, 0<y<H)
$$

Where $I_{\mu}$ is the mean vector of three channels and $I_{\omega_{h c}}(x, y)$ is the processed image after Gaussian filtering. For the Gaussian kernel, we choose a $5 \times 5$ separable binomial matrix.

The saliency map in HSI color space is calculated in the same way stated above. Afterward we can obtain a final saliency map by adding the Lab map and the HSI map together, which is shown in Fig. 3 (a). We can see from the figure that the method based on saliency map is able to separate most of red and white cells from the background. However, there still exist some other substances that have not been removed completely. In addition, it is difficult to discriminate those impurities and the cells with weak boundaries. To tackle these issues, we leverage the marker-controlled watershed algorithm based on iterative erosion for further segmentation.

With some additional experience, the marker-controlled watershed algorithm based on iterative erosion specifies some foreground and background markers and update them using traditional watershed algorithm. The foreground marker is defined inside each target area of interest, while the background marker in the other areas. The segmentation algorithm mainly includes the following steps: (1) Calculate the gradient magnitude of the original image. (2) Get the foreground marker. 8-Neighbor structural elements are used to iteratively corrode the saliency map to obtain the seed point image of the cell as the foreground marker (3) Get background marker. Euclidean distance transformation is performed on the foreground marker image. The 
watershed ridge line is obtained as the background marker using watershed segmentation. The foreground and background marker images are shown in Fig. 3(b). (4) Use the H-minima method to modify the gradient of the image so that it only has a local minimum at the foreground and background markers. (5) Watershed method for segmentation. The segmentation result is shown in Fig. 3(c). (6) Remove the tangible components which differ greatly from the red and white cells according to the areas of the connected components and the sizes of the circumscribed rectangles. The final segmentation result is shown in Fig. 3(d).

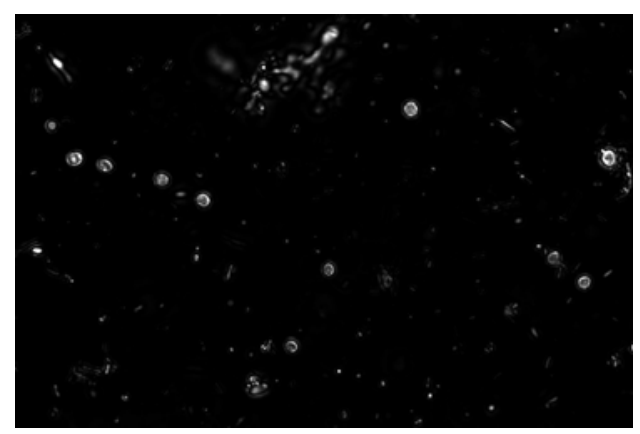

(a) The saliency map of stool microscopic image

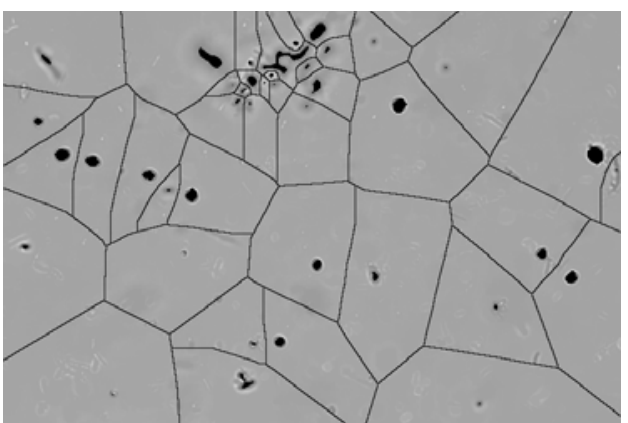

(b) The image with foreground and background markers

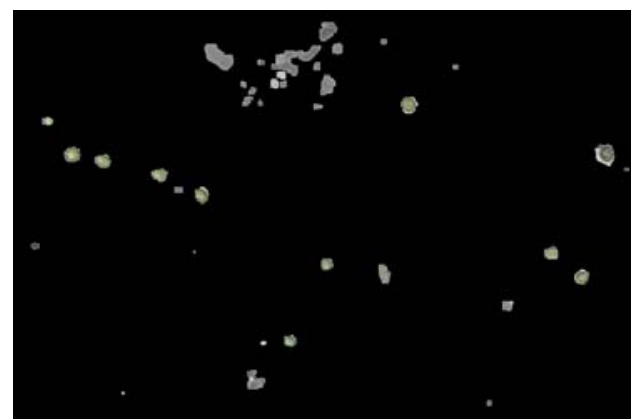

(c) The result of marker-controlled watershed segmentation

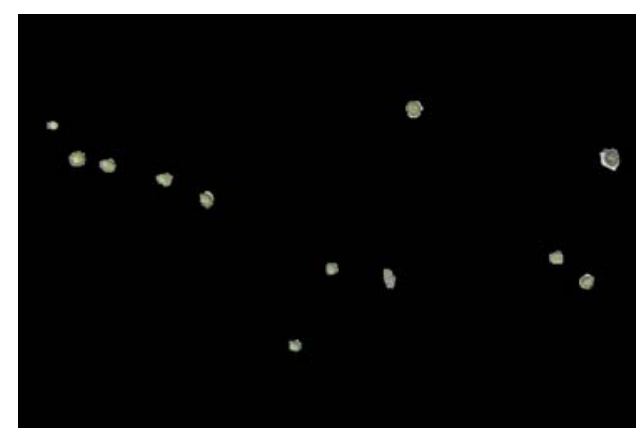

(d) The final segmented image

Fig.3. Our segmentation results of a stool microscopic image

\subsection{Feature Selection and Extraction}

The selection and extraction of cell features is the critical part for subsequent classification using SVM. It is necessary to select appropriate features to describe the red and white cells, in order to improve the accuracy of classification. We are proposed to choose some geometric features (area, perimeter and circularity) and texture features (energy, entropy, correlation and contrast) as feature vectors to train the SVM classifier. Table 1 describes our selected features and their calculation formulas. The geometric features in Table 1 are calculated based on the segmented binary image, yet the texture features are calculated based on the grey level co-occurrence matrix(GLCM). Wherein the GLCM is a matrix that is defined over an image to be the distribution of co-occurring pixel values (grayscale values, or colors) at a given offset. It is based on studies of the statistics of pixel intensity distributions. Since single pixel statistics do not provide rich enough descriptions of textures for practical applications, it is natural to consider the second-order statistics obtained by considering pairs of pixels in certain spatial relations to each other. Hence, the co-occurrence matrices are used, which express the relative frequencies (or probabilities) $P(i, j \mid d, \theta)$ with which two pixels having relative polar coordinates $(d, \theta)$ appear with intensities $i, j$. The co-occurrence matrices provide raw numerical data on the texture, although these data must be condensed to relatively few numbers before they can be used to classify the texture.

$P(i, j \mid d, \theta)$ in Table 1 represents the joint probability of grey levels $i$ and $j$ in the co-occurrence matrix when the distance between the separated pixels is $d$ and the direction is $\theta$.When calculating the texture features, we choose four different directions $\theta=0^{\circ}, 45^{\circ}, 90^{\circ}, 135^{\circ}$ to generate the co-occurrence matrix. The pixel distance $d=1$, and the image grey level is compressed to 16 to calculate the GLCM. The $\mu_{x}, \mu_{y}, \sigma_{x}, \sigma_{y}$ in the fifth formula in Table 1 are the mean values and the mean-square deviations along $X$ and $Y$ directions respectively. Fig. 4 is the flow chart which displays the steps of textural feature extraction.

Table 1. Selected Features

\begin{tabular}{ccc}
\hline Feature category & Calculation formula & Feature Description \\
\hline Area & $S=n$ & The total number of connected pixels in binary image \\
\hline
\end{tabular}




\begin{tabular}{|c|c|c|c|c|c|}
\hline Perimeter & \multicolumn{2}{|c|}{$L=\sum_{s=1}^{n} e d g e$} & \multicolumn{3}{|c|}{ Boundary length of connected regions in binary image } \\
\hline Circularity & \multicolumn{2}{|c|}{$C=4 \pi S / L^{2}$} & \multicolumn{3}{|c|}{ The similarity between the connected area and the circle in the binary image } \\
\hline Energy & \multicolumn{2}{|c|}{$E(d, \theta)=\sum_{i, j}(P(i, j \mid d, \theta))^{2}$} & \multicolumn{3}{|c|}{ Uniformity of grey distribution and texture thickness } \\
\hline Correlation & \multicolumn{2}{|c|}{$C(d, \theta)=\sum_{i, j}\left(i-\mu_{x}\right)\left(j-\mu_{y}\right) P(i, j \mid d, \theta) / \sigma_{x} \sigma_{y}$} & \multicolumn{3}{|c|}{ Texture similarity in row or column direction } \\
\hline Entropy & \multicolumn{2}{|c|}{$H(d, \theta)=-\sum_{i, j} P(i, j \mid d, \theta) \log _{2} P(i, j)$} & \multicolumn{3}{|c|}{ Texture non-uniformity or complexity } \\
\hline Contrast & \multicolumn{2}{|c|}{$I(d, \theta)=\sum(i, j)^{2} P(i, j \mid d, \theta)$} & \multicolumn{3}{|c|}{ Texture clarity and depth of grooves } \\
\hline $\begin{array}{l}\text { Sub-image after } \\
\text { segmentation }\end{array}$ & RGB $\rightarrow$ Gray & $\begin{array}{l}\text { Compressing } \\
\text { the gray level }\end{array}$ & $\begin{array}{l}\text { Calculating GLCM in } \\
\text { different directions }\end{array}$ & $\begin{array}{c}\text { Calculating texture } \\
\text { parameters }\end{array}$ & $\begin{array}{l}\text { Texture } \\
\text { features }\end{array}$ \\
\hline
\end{tabular}

Fig.4. The main procedure of textural feature extraction

\subsection{Recognition of red and white cells}

After segmentation and feature extraction, the extracted feature vectors are input into SVM for classification and recognition. Since the features are non-linearly separable, it is necessary to use a kernel function to map them into a high-dimensional space for classification. The choice of kernel function is generally related to the categories of classification tasks. Considering the number of features is relatively small, we choose the Gaussian kernel function in the construction of the classifier. The Gaussian kernel function can be described as follows

$$
K\left(x_{i}, x_{j}\right)=\exp \left(-\gamma\left\|x_{i}-x_{j}\right\|^{2}\right), \gamma>0
$$

\section{Experimental results and analysis}

In this experiment, we select 6,400 stool microscopic images (a total of 400 stool samples with 16 views per sample), all of which are unstained images provided by the relevant analytical laboratory. In these images all the red and white cells are labelled by experts. We are proposed to choose 1200 of them as our experimental dataset.

\subsection{Segmentation Experiment}

In order to verify the effectiveness of our method, we compare our results (shown in Fig. 5(b)) with the improved Chan-Vese segmentation method proposed in [6] (shown in Fig. 5(a)). We can observe that the segmentation results of the method in [6] will also include more other tangible compositions besides the red and white cells. This demonstrates that our method achieves a better segmentation performance. To further compare these two methods, we use the recall to evaluate the segmentation results. The formula for calculating the recall is as follows

$$
\operatorname{Re} \text { call }=\frac{T P}{T P+F N}
$$

where $T P$ represents the true positive samples (the red and white cells that are segmented correctly), and $F N$ is the false negative samples (the red and white cells that are not detected).

Table 2. Comparison of the results after the segmentation of the stool microscopic image

\begin{tabular}{ccccc}
\hline $\begin{array}{c}\text { Segmentation } \\
\text { method }\end{array}$ & TP & FP & FN & Recall(\%) \\
\hline Reference[6] & 3128 & 689 & 204 & 93.8 \\
Our method & 3216 & 412 & 116 & 96.5 \\
\hline
\end{tabular}

The quantitative comparison between two methods are shown in Table 2. From the table we can find that our recall is $96.5 \%$, which is much higher than [6]. The number of false positive samples of our algorithm is also smaller than that in [6], which indicates we rarely recognize red and white cells as other things, namely our method achieves a lower error rate. As a result, our method performs more accurate and robust in segmenting the red and white cells with fuzzy edges.

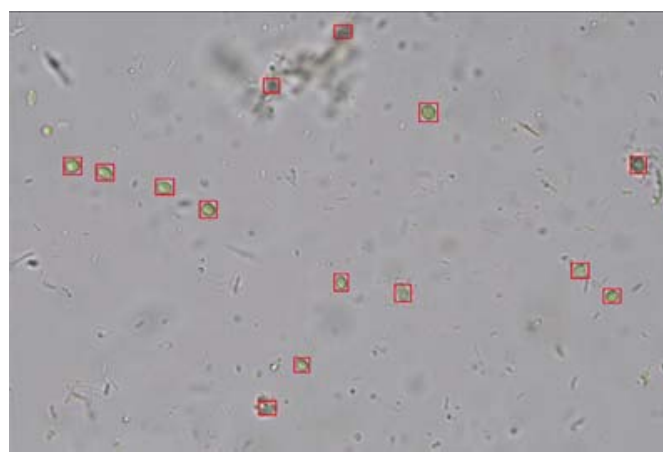

(a) Segmentation results of reference [6] 


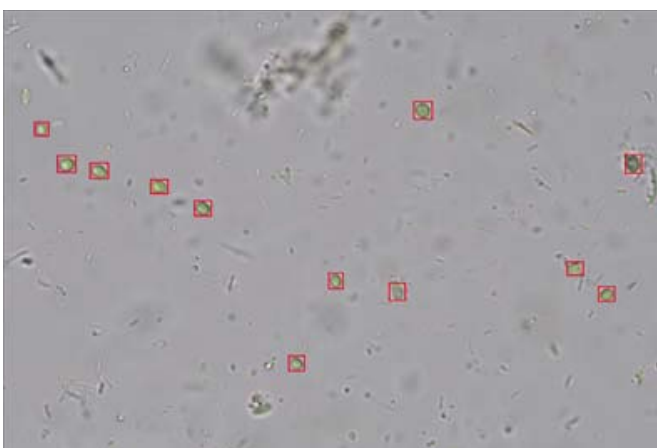

(b) Segmentation results of our algorithm

Fig.5. Segmentation results of red and white cells by different algorithms

\subsection{Recognition Experiment}

The segmented image still contains impurities which are similar to the red and white cells in morphology. In order to further improve the accuracy of cell detection and distinguish between red and white cells, we extract the geometric and texture features of the segmented samples described in Table 1 and use SVM to classify them. The recognition experiment in this paper is based on LIBSVM [14]. 400 red cells, white cells, and impurities were selected respectively from the segmented samples, and divided into a training set and a test set according to ratio $4: 1$. We select the penalty factor $C=16$ and the kernel function parameter $\gamma=0.3536$ for the Gaussian kernel using the grid search. Also, we apply the 5-fold cross validation to avoid overfitting and selection bias.

Our classification result is shown in Fig. 6. The red cells are marked with red rectangular frames, while the white cells are marked with red round frames, and the impurities are marked with black round frames. The precision $(P R E)$ and false precision $(F P R)$ are used to evaluate the recognition performance, which is defined as

$$
\begin{aligned}
& P R E=\frac{T P}{T P+F P} \\
& F P R=\frac{F P}{F P+T N}
\end{aligned}
$$

Besides the TP and FP introduced above, the $T N$ represents the true negative samples, which means the impurities which are classified correctly.

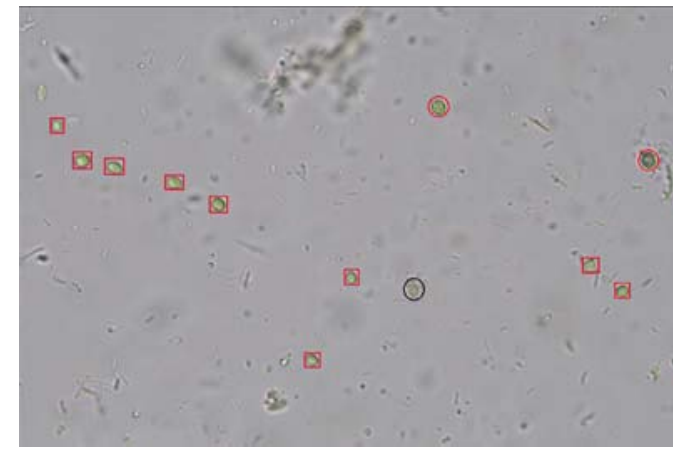

Fig.6. Our recognition result in a stool microscopic image

Table 3 compares our classification results of red and white cells with another two methods. In terms of red cells or white cells, our method achieves the best recognition performance. This illustrates it is more effective and suitable for cell recognition using SVM classifier combined with the geometry and textural features we select.

Table 3. Recognition results of different methods

\begin{tabular}{|c|c|c|c|c|}
\hline \multirow{2}{*}{$\begin{array}{c}\text { Method of } \\
\text { classification }\end{array}$} & \multicolumn{2}{|c|}{ Precision (\%) } & \multicolumn{2}{c|}{ False precision (\%) } \\
\cline { 2 - 6 } & Red cells & White cells & Red cells & White cells \\
\hline BP neural network [7] & 88.5 & 89.7 & 8.0 & 7.8 \\
\hline Random forest [6] & 94.9 & 95.3 & 4.1 & 3.9 \\
\hline Our method & 96.1 & 95.9 & 3.8 & 3.5 \\
\hline
\end{tabular}

\section{Conclusion}

Aiming at the problem of blurred boundary of cells in stool microscopic images, we propose a new segmentation and recognition method to improve the accuracy of detecting red and white cells. For cell segmentation, we introduce an approach which combines the saliency map with the marker-controlled watershed segmentation based on iterative corrosion. For cell recognition, we apply the SVM classifier trained with series of appropriate and effective geometry and textural features. Experimental results show that our method achieves a much better performance for segmentation and recognition of cells in stool microscopy images than past approaches. Our research realizes the automatic segmentation and recognition of red and white cells with high precision, which can reduce the workload of artificial cell detection to a certain extent. We expect our method can be further applied in clinical practice in the future.

\section{References}

1. D. Tchiotsop, et al, "Edge detection of intestinal parasites in stool microscopic images using multi-scale wavelet transform," Signal, Image and Video Processing, vol. 9, pp. 121-134, (2015)

2. C. Sommer and D. W. Gerlich, "Machine learning in cell biology - teaching computers to recognize phenotypes," J CELL SCI, vol. 126, pp. 5529-5539, (2013)

3. Y. Pan, T. Zhou and Y. Xia, "Bacterial foraging based edge detection for cell image segmentation,", United States, pp. 3873-3876, (2015)

4. J. CHALFOUN, et al, "Empirical gradient threshold technique for automated segmentation across image modalities and cell lines," J MICROSC-OXFORD, vol. 260, pp. 86-99, (2015)

5. Ya W. Adaptive marked watershed segmentation algorithm for red blood cell images[J]. Journal of Image and Graphics, (2017) 
6. Xiangang J, Xiaoling H E, Zizhu F, et al. Methods of segmentation and recognition for red and white cells in stool microscopy images $[\mathrm{J}]$. Computer Engineering and Applications, (2018)

7. Wang Liping, Liu Renren. Red blood cell recognition based on BP neural network [J]. Computer and Digital Engineering, 46 (03): 584-587, (2018)

8. Molina-Cabello M A, Ezequiel López-Rubio, Luque-Baena R M, et al. Blood Cell Classification Using the Hough Transform and Convolutional Neural Networks[C]. World Conference on Information Systems \& Technologies. Springer, Cham (2018)

9. R. Achanta, S. Hemami, F. Estrada and S. Susstrunk, "Frequency-tuned salient region detection," CVPR, Miami, FL, 2009, pp. 1597-1604.

10. P. Soille, "Morphological carving," PATTERN RECOGN LETT, vol. 25, pp. 543-550, (2004)

11. Chen Junbo, Yu Shenghui, Shu Zhenyu. Medical image segmentation based on control marker watershed [J]. Journal of Yangtze University (Natural Science Edition) Science and Technology, 2008 (02): 96-98 + 141.

12. Wang Wei, Si Miaomiao, Chen Fuhui, et al. Study on the identification method of label-free red and white blood cells in low magnification microscopic images [J]. Journal of Chongqing University of Posts and Telecommunications: Natural Science Edition, 2019 (4): 578-584.

13. S. M. Erfani, S. Rajasegarar, S. Karunasekera, and C. Leckie, "High-Dimensional and Large-Scale Anomaly Detection using a Linear One-Class SVM with Deep Learning," PATTERN RECOGN, p. S0031320316300267, (2016)

14. Chih-Chung, Chang, Chih-Jen, et al. LIBSVM: A library for support vector machines[J]. Acm Transactions on Intelligent Systems \& Technology, (2011) 